\title{
Effectiveness of aluminosilicate-based products for detoxification of aflatoxin-contaminated diets for juvenile Pacific white shrimp, Litopenaeus vannamei
}

\section{Efectividad de productos a base de aluminosilicatos en la detoxificación de dietas contaminadas con aflatoxinas para juveniles de camarón blanco del Pacífico, Litopenaeus vannamei}

\author{
Oscar Daniel García-Pérez, Mireya Tapia-Salazar*, Martha Guadalupe Nieto-López, David Villarreal- \\ Cavazos, Lucía Elizabeth Cruz-Suárez, Denis Ricque-Marie
}

Programa Maricultura, Facultad de Ciencias Biológicas, Universidad Autónoma de Nuevo León, Ciudad Universitaria, San Nicolás de los Garza, Nuevo León 66450, México.

* Corresponding author. E-mail: mireya.tapia@gmail.com, mireya.tapias1@uanl.edu.mx

\begin{abstract}
The safety and effectiveness of four aluminosilicate-based products (Novasil Plus, Zeolex Extra, Mycofix Select, and Fixat) were tested for 42 days on juvenile Pacific white shrimp, Litopenaeus vannamei, fed diets containing corn grains that had been previously inoculated with Aspergillus parasiticus and then sterilized. Two control diets, one uncontaminated and another contaminated with the contaminated corn grain, were elaborated to contain a final concentration of 0 and $75 \mu \mathrm{g} \mathrm{kg} \mathrm{k}^{-1}$ total aflatoxins. Both diets were formulated to contain $38 \%$ crude protein and $8 \%$ lipids. Eight more diets were manufactured from the previous two to contain each of the test products at inclusion levels of 2.5 to $5.0 \mathrm{~g} \mathrm{~kg}^{-1}$, in accordance with the inclusion level recommended by the manufacturer. Mycofix Select and Fixat inclusion improved diet stability (12-16\%). Water absorption capacity in the diets increased (from 2\% to 17\%) due to the supplementation of aluminosilicates. Feeding aflatoxin-contaminated diets to Pacific white shrimp resulted in lower feed intake and weight gain without affecting the feed conversion ratio, nitrogen retention, and survival. Feeding the uncontaminated diets supplemented with Zeolex Extra, Mycofix Select, and Fixat resulted in a slight reduction in feed intake without affecting growth rate, nitrogen retention, or survival. Conversely, the inclusion of Novasil Plus in the uncontaminated diet did not affect feed intake and an $8 \%$ growth rate improval was observed. Three of the tested products failed to provide complete restoration of feed consumption and shrimp growth rate when added to the aflatoxin-contaminated diet.
\end{abstract}

Key words: aluminosilicates, shrimp, aflatoxin.

RESUMEN. Se evaluaron la seguridad y efectividad de cuatro productos a base de aluminosilicatos (Novasil Plus, Zeolex Extra, Mycofix Select y Fixat) durante 42 días en juveniles de camarón blanco del Pacífico, Litopenaeus vannamei, alimentados con granos de maíz que habían sido previamente inoculados con Aspergillus parasiticus y después esterilizados. Dos dietas control, una no contaminada y otra contaminada con el grano de maíz inoculado, fueron elaboradas para contener una concentración de 0 y $75 \mu \mathrm{g} \mathrm{kg}^{-1}$ de aflatoxinas totales. Ambas dietas fueron formuladas para contener $38 \%$ de proteína cruda y $8 \%$ de lípidos crudos. Ocho dietas más fueron elaboradas a partir de las dos dietas controles para contener cada uno de los productos a evaluar a un nivel de inclusión de 2.5 a $5.0 \mathrm{~g} \mathrm{~kg}^{-1}$, siguiendo las recomendaciones del productor. La inclusión de Mycofix Select y Fixat mejoró la estabilidad de las dietas (12-16\%). La capacidad de absorción de agua de las dietas se incrementó (de $2 \%$ a 17\%) debido a la suplementación de aluminosilicatos. Las dietas contaminadas con aflatoxinas disminuyeron el consumo de alimento y el aumento de peso sin afectar la tasa de conversión alimenticia, la eficiencia de retención de nitrógeno y la supervivencia de los camarones. La alimentación con las dietas no contaminadas suplementadas con Zeolex Extra, Mycofix Select y Fixat redujo ligeramente el consumo de alimento sin decremento de la tasa de crecimiento, la eficiencia de retención de nitrógeno y la supervivencia. En cambio, la inclusión de Novasil Plus en la dieta no contaminada no afectó el consumo de alimento y mejoró la tasa de crecimiento en un $8 \%$. Tres de los productos evaluados no proporcionaron una restauración completa del consumo de alimento y la tasa de crecimiento cuando fueron suplementados a una dieta contaminada con aflatoxinas.

Palabras clave: aluminosilicatos, camarón, aflatoxinas.

\section{INTRODUCTION}

Mycotoxins are secondary metabolites produced by fungi, mainly of the genera Aspergillus, Penicillium, Fusarium, and Alternaria, and may be found in feedstuffs or feeds (Kolossova et al. 2009). An important reduction in feed

\section{INTRODUCCIÓN}

Las micotoxinas son metabolitos secundarios producidos por hongos, especialmente de los géneros Aspergillus, Penicillium, Fusarium y Alternaria, y se pueden encontrar en forrajes y alimentos (Kolossova et al. 2009). Se ha observado 
intake, depression of growth performance, immune suppression, and hepatic lesions have been observed in rainbow trout (Arana et al. 2011), carp (Mohapatra et al. 2011), tilapia (Shehata et al. 2009), catfish (Döll et al. 2010), and shrimp (Boonyaratpalin et al. 2001, Soonngam and Hutacharoen 2007, Gopinath and Raj 2009, Tapia-Salazar et al. 2012) fed an aflatoxin-contaminated diet. Several detoxifying products are commercially available to inactivate mycotoxins in animal feeds, and their detoxifying effect involves physical adsorption, binding, and trapping of toxic substances (Kolossova et al. 2009), followed by their elimination together with the detoxifier. In vitro and in vivo studies have shown that hydrated sodium calcium alumino-silicates (HSCAS, Döll et al. 2005), montmorillonites (Ramos and Hernández 1996), bentonites (Schell et al. 2000), and zeolites (Piva et al. 1995) have a high binding affinity for aflatoxins. Two studies using aluminosilicates in diets for Pacific white shrimp, Litopenaeus vannamei, failed to demonstrate the elimination of negative effects from aflatoxins (Suppadit $e t$ al. 2006). The same was reported for the black tiger shrimp, Penaeus monodon (Soonngam and Hutacharoen 2007). The aim of the present study was to evaluate the effectiveness and safety of four commercial aluminosilicate products to reduce the effect of aflatoxins contained in diets offered juvenile Pacific white shrimp.

\section{MATERIAL AND METHODS}

\section{Inoculation of corn with fungus}

A contaminated corn sample was provided by Nutek (Tehuacán, Puebla, Mexico), which had been prepared as follows. Briefly, a fungal inoculum was grown from singlespore cultures of Aspergillus parasiticus for five days at $25^{\circ} \mathrm{C}$ in commercial potato-dextrose agar (plate), titrated and diluted to a concentration of $1 \times 10^{6}$ spores $\mathrm{mL}^{-1}$. Grains were incubated with the A. parasiticus inoculum for 7 days at $28^{\circ} \mathrm{C}$. The inoculated corn grains were then sterilized for $30 \mathrm{~min}$ at $121^{\circ} \mathrm{C}$, dried for four days at room temperature with air circulation, and ground to a mean particle size of $850 \mu \mathrm{m}$ (mesh \#20).

\section{Aluminosilicate-based products}

The following test products were used: Novasil Plus (HSCAS, Ca-montmorillonite, 62.4-73.5\% $\quad \mathrm{SiO}_{2}$, $14.8-18.2 \% \quad \mathrm{Al}_{2} \mathrm{O}_{3}, \quad 3.2-4.8 \% \quad \mathrm{CaO}, \quad 4.0-4.5 \% \quad \mathrm{MgO}$, 5.4-6.5\% $\quad \mathrm{Fe}_{2} \mathrm{O}_{3}, \quad 0.5-0.9 \% \quad \mathrm{~K}_{2} \mathrm{O}, \quad 0.1-0.3 \% \quad \mathrm{Na}_{2} \mathrm{O}$, $0.01-0.03 \% \mathrm{MnO}$ ), Zeolex Extra (chemically activated organoaluminosilicate, $\quad 57-64 \% \quad \mathrm{SiO}_{2}, \quad 10-17 \% \quad \mathrm{Al}_{2} \mathrm{O}_{3}$, 3.3-4.9\% CaO, 0.3-1.8\% MgO, 0.6-1.4\% $\mathrm{Fe}_{2} \mathrm{O}_{3}, 1.8-2.8 \%$ $\mathrm{K}_{2} \mathrm{O}$, 1.6-2.9\% $\mathrm{Na}_{2} \mathrm{O}$ ), Mycofix Select (diatomaceous earth $30 \%$, kaolinite $50 \%$, phytophytic substances $10 \%$, herbal extracts 10\%), and Fixat (bentonite-montmorillonite, $50-65 \% \mathrm{SiO}_{2}, 15-25 \% \mathrm{Al}_{2} \mathrm{O}_{3}, 3-6 \% \mathrm{CaO}, 3-6 \% \mathrm{MgO}, 3-6 \%$ una reducción notable del consumo de alimento, disminución de crecimiento, supresión del sistema inmunológico y lesiones hepáticas en truchas arcoiris (Arana et al. 2011), carpas (Mohapatra et al. 2011), tilapias (Shehata et al. 2009), bagres (Döll et al. 2010) y camarones (Boonyaratpalin et al. 2001, Soonngam y Hutacharoen 2007, Gopinath y Raj 2009, TapiaSalazar et al. 2012) alimentados con dietas contaminadas con aflatoxinas. Existen varios productos de desintoxicación comerciales para inactivar las micotoxinas en los alimentos para animales y su efecto desintoxicante consiste en la adsorción física, unión y secuestro de sustancias tóxicas (Kolossova et al. 2009), que después son eliminadas junto con el desintoxicante. En estudios in vitro e in vivo se ha mostrado que los aluminosilicatos de calcio y sodio hidratados (HSCAS por sus siglas en inglés, Döll et al. 2005), las montmorillonitas (Ramos y Hernández 1996), las bentonitas (Schell et al. 2000) y las zeolitas (Piva et al. 1995) tienen una alta afinidad por las aflatoxinas. Dos estudios que usaron aluminosilicatos en dietas para el camarón blanco del Pacífico, Litopenaeus vannamei, no pudieron demostrar la eliminación de los efectos negativos de las aflatoxinas (Suppadit et al. 2006). Lo mismo se documentó para el camarón tigre, Penaeus monodon (Soonngam y Hutacharoen 2007). El objetivo del presente trabajo fue evaluar la efectividad y seguridad de cuatro aluminosilicatos comerciales para reducir el efecto de las aflatoxinas en dietas proporcionadas a juveniles de camarón blanco del Pacífico.

\section{MATERIALES Y MÉTODOS}

\section{Inoculación del maíz con hongo}

La empresa Nutek (Tehuacán, Puebla, México) proporcionó una muestra de maíz contaminado, preparado de la siguiente manera. De forma resumida, se preparó un inóculo fúngico a partir de cultivos de esporas únicas de Aspergillus parasiticus, se cultivó durante cinco días a $25^{\circ} \mathrm{C}$ en un medio de agar papa dextrosa comercial (placa) y se tituló y diluyó hasta obtener $1 \times 10^{6}$ esporas $\mathrm{mL}^{-1}$. Los granos se incubaron con el inóculo de A. parasiticus durante 7 días a $28^{\circ} \mathrm{C}$. Posteriormente, los granos de maíz inoculados se esterilizaron durante $30 \mathrm{~min}$ a $121^{\circ} \mathrm{C}$, se dejaron secar durante cuatro días a temperatura ambiente con circulación de aire y se molieron a un tamaño de partícula promedio de $850 \mu \mathrm{m}$ (malla \#20).

\section{Productos a base de aluminosilicatos}

Se utilizaron los siguientes productos en las dietas experimentales: Novasil Plus (HSCAS, montmorillonita de $\mathrm{Ca}, 62.4-73.5 \% \mathrm{SiO}_{2}, 14.8-18.2 \% \mathrm{Al}_{2} \mathrm{O}_{3}, 3.2-4.8 \% \mathrm{CaO}$, 4.0-4.5\% MgO, 5.4-6.5\% $\mathrm{Fe}_{2} \mathrm{O}_{3}, 0.5-0.9 \% \mathrm{~K}_{2} \mathrm{O}, 0.1-0.3 \%$ $\mathrm{Na}_{2} \mathrm{O}, 0.01-0.03 \% \mathrm{MnO}$ ), Zeolex Extra (organoaluminosilicato químicamente activado, 57-64\% $\mathrm{SiO}_{2}, 10-17 \% \mathrm{Al}_{2} \mathrm{O}_{3}$, $3.3-4.9 \% \mathrm{CaO}, 0.3-1.8 \% \mathrm{MgO}, 0.6-1.4 \% \mathrm{Fe}_{2} \mathrm{O}_{3}, 1.8-2.8 \%$ $\mathrm{K}_{2} \mathrm{O}, 1.6-2.9 \% \mathrm{Na}_{2} \mathrm{O}$ ), Mycofix Select (tierra de diatomeas 
$\mathrm{Fe}_{2} \mathrm{O}_{3},<5 \% \mathrm{Na}_{2} \mathrm{O}$ and $\mathrm{K}_{2} \mathrm{O}$ ). Aluminosilicate inclusion levels ranged from 2.5 to $5.0 \mathrm{~g} \mathrm{~kg}^{-1}$ depending on the inclusion level recommended for each test product (table 1).

\section{Experimental diets}

\section{Formulas}

A contaminated diet (CD) and a non-contaminated diet (NCD) were formulated to contain $38 \%$ crude protein and $8 \%$ crude lipids. In $\mathrm{CD}$, the contaminated corn was included at a level of $11.1 \%$, mainly at the expense of wheat meal, to obtain $75 \mu \mathrm{g} \mathrm{kg}^{-1}$ total dietary aflatoxins. From these two control diets, eight other diets were manufactured to contain one of the commercial products, in substitution of wheat meal (table 1).

Preparation, chemical composition, stability, percentage of water absorption, and mycotoxin analysis of the test diets

The ingredients were ground in a Cyclotec grinder (Tecator, model 1093) to obtain a mean particle size of $500 \mu \mathrm{m}$. Ingredients were mixed for $10 \mathrm{~min}$ in a Kitchen Aid mixer; water $(30 \%)$ was added and mixed for a further $15 \mathrm{~min}$. The wet diet mash was passed through a meat grinder (fitted with a die having $1.6 \mathrm{~mm}$ diameter holes) at a rate of $40 \mathrm{~min} \mathrm{~kg}^{-1}$ diet and reaching a temperature of $70-75^{\circ} \mathrm{C}$. The spaghetti-like strands were dried in a convection oven at $100{ }^{\circ} \mathrm{C}$ for $8 \mathrm{~min}$ and allowed to cool and dry overnight at room temperature before packing. Chemical analysis to determine proximate composition of the ingredients and test diets was conducted following the standard procedure. Aflatoxin concentrations (B1, B2, G1, and G2) in the test diets were analyzed by high-performance liquid chromatography at Trilogy Analytical Laboratory, Washington, MO. The control diets were also analyzed for deoxynivalenol, fumonisins (B1, B2, and B3), ochratoxin A, and T-2 toxin. To determine the percentage of dry matter loss, a $3 \mathrm{~g}$ pellet sample was weighed and placed over a sieve (\#40 mesh), and hung inside a plastic bottle containing $200 \mathrm{~mL}$ of seawater; the bottles were shaken inside a water bath for $1 \mathrm{~h}\left(30 \mathrm{rpm}, 28^{\circ} \mathrm{C}\right)$, and the sieves were then drained for a few minutes and dried at constant weight: dry matter loss $(\%)=100 \times[\mathrm{DWd}-$ DWwid $+($ WWwid - DWwid $) \times$ sal] $/$ DWd, where DWd and DWwid are the dry weights of the diet sample before and after the water immersion, respectively, WWwid is the wet weight of the diet sample after immersion, and sal is the weight of total salts per gram of seawater. To determine water absorption in the experimental diets, a $5 \mathrm{~g}$ sample of feed was weighed and submerged for $1 \mathrm{~h}$ in $50 \mathrm{~mL}$ of distilled water; the wet sample was filtered through a \#200 mesh screen and the retained sample was weighed: water absorption (\%) = $100 \times[$ (weight of the sample after submersion in distilled water - weight of the sample before submersion)/weight of the sample before submersion] $\times 100$.
$30 \%$, caolinita $50 \%$, sustancias fitofíticas $10 \%$, extractos herbales 10\%), y Fixat (bentonita montmorillonita, 50-65\% $\mathrm{SiO}_{2}, 15-25 \% \mathrm{Al}_{2} \mathrm{O}_{3}, 3-6 \% \mathrm{CaO}, 3-6 \% \mathrm{MgO}, 3-6 \% \mathrm{Fe}_{2} \mathrm{O}_{3}$, $<5 \% \mathrm{Na}_{2} \mathrm{O}$ y $\mathrm{K}_{2} \mathrm{O}$ ). Los niveles de inclusión de los aluminosilicatos variaron de 2.5 a $5.0 \mathrm{~g} \mathrm{~kg}^{-1}$ según los niveles recomendados para cada producto (tabla 1 ).

\section{Dietas experimentales}

\section{Fórmulas}

Se formuló una dieta contaminada (CD) y una dieta no contaminada (NCD) para contener $38 \%$ de proteína cruda y $8 \%$ de lípidos crudos. En la CD, el maíz contaminado se incluyó a un nivel de $11.1 \%$, principalmente a expensas de harina de trigo, para obtener $75 \mu \mathrm{g} \mathrm{kg}^{-1}$ de aflatoxinas totales en la dieta. A partir de estas dos dietas controles, se elaboraron otras ocho dietas para contener uno de los productos comerciales, en sustitución de harina de trigo (tabla 1).

Preparación, composición química, estabilidad, porcentaje de absorción de agua y análisis de micotoxinas de las dietas experimentales

Los ingredientes fueron molidos en un molino Cyclotec (Tecator, modelo 1093) hasta obtener un tamaño de partícula promedio de $500 \mu \mathrm{m}$. Los ingredientes se mezclaron durante $10 \mathrm{~min}$ en una mezcladora Kitchen Aid; se agregó agua (30\%) y se continuó mezclando durante $15 \mathrm{~min}$. La mezcla húmeda se pasó por un molino de carne (equipado con un dado con orificios de $1.6 \mathrm{~mm}$ de diámetro) a una tasa de 40 min $\mathrm{kg}^{-1}$ y hasta alcanzar una temperatura de $70-75^{\circ} \mathrm{C}$. Las tiras en forma de espagueti fueron secadas en un horno de convección a $100{ }^{\circ} \mathrm{C}$ durante 8 min y se dejaron enfriar y secar durante la noche a temperatura ambiente antes de empaquetarlas. El análisis químico para determinar la composición proximal de los ingredientes y las dietas experimentales se realizó siguiendo el procedimiento estándar. Las concentraciones de aflatoxinas (B1, B2, G1 y G2) en las pruebas experimentales se analizaron mediante cromatografía líquida de alta eficacia en Trilogy Analytical Laboratory, Washington, MO. Las dietas control también fueron analizadas para deoxinivalenol, fumonisinas (B1, B2 y B3), ocratoxina A y toxina T-2. Para determinar el porcentaje de pérdida de materia seca, una muestra de $3 \mathrm{~g}$ de alimento se pesó y colocó sobre un tamiz (malla \#40), y se colgó dentro de una botella de plástico con $200 \mathrm{~mL}$ de agua de mar; después de agitar las botellas dentro de un baño de agua durante $1 \mathrm{~h}\left(30 \mathrm{rpm}, 28^{\circ} \mathrm{C}\right)$, los tamices se retiraron, se dejaron escurrir durante unos minutos y se pesaron a peso constante: pérdidad de materia seca $(\%)=100 \times[\mathrm{PSd}-$ PSdía + (PHdía - PSdía $) \times$ sal] $/$ PSd, donde PSd y PSdía son el peso seco de la muestra de dieta antes y después, respectivamente, de la inmersión en agua, PHdía es el peso húmedo de la dieta 


\section{Growth trial}

A feeding trial was carried out in a closed recirculation system holding artificial seawater. The experimental facility consisted of 60-L fiberglass tanks, each tank continuously fed with synthetic marine water (Fritz, Dallas, TX) at a flow-

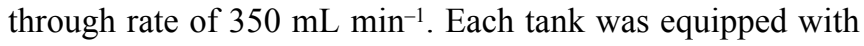
an air lift water system for internal recirculation. The facility was designed so that possible water quality variations affect all tanks simultaneously. Salinity and temperature were measured daily, while $\mathrm{pH}$, total ammonia, nitrites, and nitrates were recorded weekly. Water quality parameters were: salinity, 32-37; temperature, $27-30^{\circ} \mathrm{C}$; $\mathrm{pH}, 8.0-8.2$; total

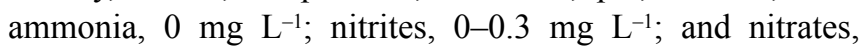
$50 \mathrm{mg} \mathrm{L}^{-1}$. The parameters remained within the optimum values for $L$. vannamei throughout the trial. Juvenile L. vannamei were obtained from a shrimp hatchery (Laboratorio de Langostinos y Camarones, Boca del Río, Veracruz, Mexico), and were acclimated to the conditions of the bioassay facilities in 500-L holding tanks prior to the growth trial. Each diet was evaluated in three replicate tanks, each holding 10 shrimp with a mean initial weight of $592 \pm 60 \mathrm{mg}$. Shrimp were individually weighed on a digital balance after blotting off excess water with a moist cloth. Care was taken to distribute animals with the same size distribution pattern in each tank. Dietary treatments were then randomly assigned to the tanks. Over the first three days after distributing the animals, any dead shrimp was replaced from a pool of animals fed the same dietary treatment. A photoperiod was set up to provide $12 \mathrm{~h}$ light and $12 \mathrm{~h}$ dark.

\section{Feeding protocol}

Initial feeding ratio was $10 \%$ of the biomass of each tank, and uneaten feed was removed by siphoning before feeding. Feeding rate was determined using feeding trays. The weighed strands of feed were broken into small pieces to ensure a minimum of one pellet per shrimp at each feeding. Shrimp were fed twice at day $(50 \%$ of the ration at 9:00 and 17:00) for 42 days.

\section{Zootechnical parameters}

Shrimp were weighed by tank once every week. Percentage weight gain, feed intake, feed conversion ratio, and survival rate were calculated weekly. Individual weights were taken at $0,14,28$, and 42 days; shrimp were weighed on a digital balance $( \pm 1.0 \mathrm{mg})$ after having been blotted dry with a cloth. Percentage weight gain was calculated, per tank, as the difference in weight from the average initial weight: weight gain $=[$ (average individual final weight - average individual initial weight) / average individual initial weight] $\times 100$. The survival rate was calculated for each tank as follows: (final number/initial number) $\times 100$. Feed consumption was estimated every day from the feed added to the tank, the feed después de la inmersión en agua y sal es el peso del total de sales por gramo de agua de mar. Para determinar la absorción de agua en las dietas experimentales, una muestra de $5 \mathrm{~g}$ de alimento se pesó y se sumergió durante $1 \mathrm{~h}$ en $50 \mathrm{~mL}$ de agua destilada, después del cual se filtró a través de una malla \#200 y se pesó: absorción de agua $(\%)=100 \times[$ (peso de la muestra después de sumersión en agua destilada - peso de la muestra antes de sumersión)/peso de la muestra antes de sumersión] $\times 100$.

\section{Bioensayo de crecimiento}

Se realizó un bioensayo en un sistema circulatorio cerrado con agua de mar artificial. El sistema experimental consistió de tanques de fibra de vidrio de $60 \mathrm{~L}$, cada uno alimentado continuamente con agua marina sintética (Fritz,

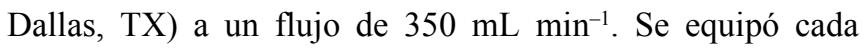
tanque con un sistema de recirculación de agua interna. El sistema fue diseñado para que las variaciones en la calidad del agua afectaran todos los tanques simultáneamente. La salinidad y la temperatura fueron medidos diariamente, mientras que el $\mathrm{pH}$, el amoniaco total y los nitritos y nitratos fueron registrados semanalmente. Los parámetros de la calidad del agua fueron: salinidad, 32-37; temperatura, $27-30^{\circ} \mathrm{C}$; $\mathrm{pH}, 8.0-8.2$; amoniaco total, $0 \mathrm{mg} \mathrm{L}^{-1}$; nitritos, $0-0.3 \mathrm{mg} \mathrm{L}^{-1}$; y nitratos, $50 \mathrm{mg} \mathrm{L}^{-1}$. Los parámetros se mantuvieron dentro de los valores óptimos para $L$. vannamei durante todo el bioensayo. Los juveniles de L. vannamei se obtuvieron del Laboratorio de Langostinos y Camarones, Boca del Río, Veracruz, México, y se aclimataron a las condiciones del sistema utilizado para los bioensayos en tanques de $500 \mathrm{~L}$ antes del experimento. Cada dieta se evaluó en tres tanques (réplicas), cada uno de los cuales contenía 10 camarones con un peso promedio inicial de $592 \pm 60 \mathrm{mg}$. Los camarones se pesaron individualmente en una balanza digital después de haber sido secados con una tela húmeda. Se tuvo cuidado de distribuir los animales con el mismo patrón de talla en cada tanque. Los tratamientos dietéticos fueron asignados a los tanques al azar. En los tres primeros días después de haber distribuido los animales, los camarones muertos fueron reemplazados con otros alimentados con el mismo tratamiento. El fotoperiodo fue de $12 \mathrm{~h}$ luz y $12 \mathrm{~h}$ oscuridad.

\section{Protocolo de alimentación}

La razón de alimentación inicial fue de $10 \%$ de la biomasa de cada tanque, retirándose el alimento no consumido con un sifón antes de proporcionar el alimento. La tasa de alimentación se determinó usando bandejas de alimentación. Las tiras de alimento se pesaron y se rompieron en pedazos pequeños para asegurar el suministro de un mínimo de un pellet por camarón por vez. Los camarones fueron alimentados dos veces al día (50\% de la ración a las 9:00 y 17:00) durante 42 días. 


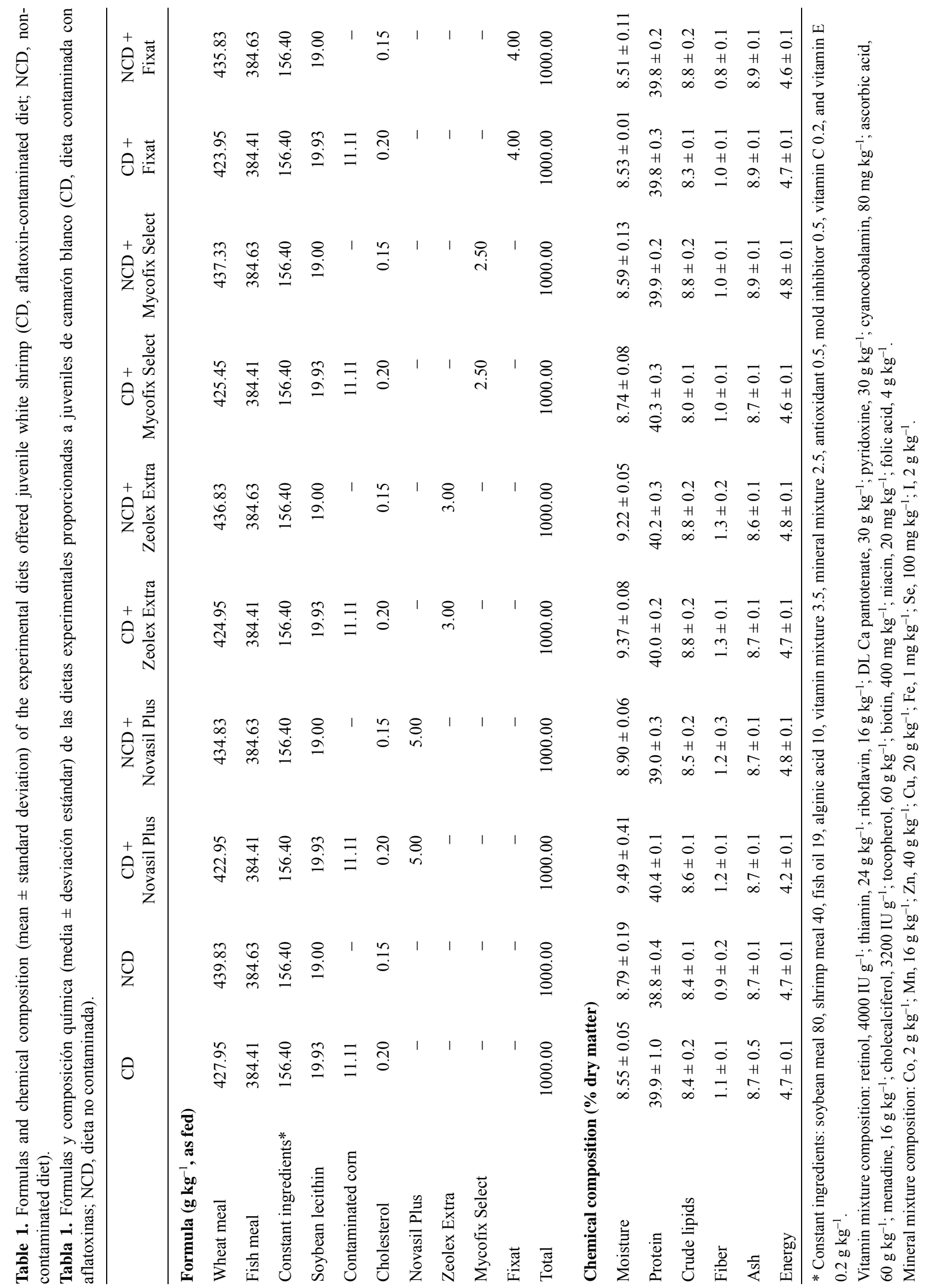


remaining the next day, and the number of shrimp in the tank; for each tank the feed consumption reported was the total of the consumption estimated for the considered period: individual consumption $=\sum_{42}^{1}$ (consumption on the $n$th day $/$ number of shrimp on the $n$th day). The feed conversion ratio is the weight of feed consumed per unit of weight gain: feed conversion ratio $=$ estimated individual consumption/average individual increase in weight. At the beginning of the experiment, a pooled sample of 60 shrimp was taken to determine the initial moisture and nitrogen composition. At the end of the experiment, 5 shrimp per tank were randomly sampled for final moisture and nitrogen content. Shrimp used for moisture and nitrogen composition were freeze-dried, ground in a coffee grinder, and stored until analysis: nitrogen retention efficiency $=[(($ final average body weight $(\mathrm{g}) \times$ crude protein content in carcass $(\%)$ ) - (initial average body weight $(\mathrm{g}) \times$ crude protein content in carcass $(\%)) /$ crude protein consumed $(\%)]$.

\section{Statistical analysis}

The results are presented as means \pm standard deviation. Individual weights were submitted to analysis of variance (ANOVA) to test for homogenous replicate means for each treatment. Dry matter loss, water absorption capacity, final weight, feed intake, growth rate, feed conversion ratio, survival, and nitrogen retention efficiency were analyzed using a $2 \times 5$ factorial ANOVA to test for the effects of the presence or absence of dietary aflatoxins, the effects of the different aluminosilicates, and the interaction of these two factors. Since interaction was significant for a number of parameters, results were also submitted to one-way ANOVA among experimental diets, followed by Tukey's multiple range tests ( $\alpha=0.05$, SSPS 16.0, 2007, SPSS Inc., Chicago, Illinois).

\section{RESUlts}

\section{Experimental diets}

The chemical composition of the test diets was similar among treatments (table 1). Aflatoxin concentration in CD was about $75 \mu \mathrm{g} \mathrm{kg}^{-1}$ and the predominant aflatoxin was B1 $\left(60.7 \mu \mathrm{g} \mathrm{kg}^{-1}\right)$, followed by B2 $\left(9.7 \mu \mathrm{g} \mathrm{kg}^{-1}\right)$ and $\mathrm{G} 1$ $\left(4.3 \mu \mathrm{g} \mathrm{kg}^{-1}\right)$. Mycotoxin concentration in NCD was below the detection limit. Deoxynivalenol, fumonisin (B1, B2, and B3), ochratoxin A, and T-2 toxin concentrations in CDs remained below the detection limit.

Dry matter loss and water absorption capacity of the test diets are presented in table 2. Significant differences due to contamination $(P=0.000)$, test products $(P=0.000)$, and interaction $(P=0.000)$ were observed for water absorption. Since interaction was significant, the interpretation of the main effects was not considered and a one-way ANOVA was carried out among treatments; results show that aluminosilicates increased water absorption, especially in $\mathrm{CDs}$, the

\section{Parámetros zootécnicos}

Los camarones se pesaron por tanque una vez por semana. El porcentaje de aumento de peso, el consumo de alimento, la tasa de conversión alimenticia y la tasa de supervivencia se calcularon semanalmente. Los días $0,14,28$ y 42, los camarones fueron pesados individualmente en una balanza digital $( \pm 1.0 \mathrm{mg})$, después de haber sido secados con una tela. El porcentaje de aumento de peso se calculó, por tanque, como la diferencia de peso en comparación con el peso inicial promedio: aumento de peso $=[($ peso final individual promedio - peso inicial individual promedio)/peso inicial individual promedio] $\times 100$. La tasa de supervivencia se calculó, por tanque, como sigue: (número final/número inicial) $\times 100$. El consumo de alimento se estimó cada día con base en el alimento introducido en cada tanque, el alimento restante al día siguiente y el número de camarones en el tanque; para cada tanque el consumo de alimento fue el total del consumo estimado para el periodo considerado: consumo individual $=\sum_{42}^{1}$ (consumo al nésimo día $/$ número de camarones al nésimo día). La tasa de conversión alimenticia es el peso del alimento consumido por unidad de aumento de peso: tasa de conversión alimenticia = consumo individual estimado/aumento de peso individual promedio. Al principio del experimento, se tomó una muestra conjunta de 60 camarones para determinar el contenido de nitrógeno y la humedad inicial. Al final del experimento, se examinaron aleatoriamente 5 camarones por tanque para determinar el contenido de nitrógeno y humedad final. Los camarones usados para determinar esto último se liofilizaron, se molieron en una moledora de café y se almacenaron hasta ser analizados: eficiencia de retención de nitrógeno $=[(($ peso corporal promedio final $(\mathrm{g}) \times$ contenido de proteína cruda corporal $(\%))-($ peso corporal promedio inicial $(\mathrm{g}) \times$ contenido de proteína cruda corporal (\%))/proteína cruda consumida (\%)].

\section{Análisis estadístico}

Los resultados se presentan como medias \pm desviación estándar. Los pesos individuales fueron sometidos a un analisis de varianza para comprobar la homogeneidad de los pesos promedio de las replicas de cada tratamiento. La pérdida de materia seca, la capacidad de absorción de agua, el peso final, el consumo de alimento, la tasa de crecimiento, la tasa de conversión alimenticia y la eficiencia de retención de nitrógeno se analizaron mediante un análisis de varianza factorial $2 \times 5$ para evaluar los efectos de la presencia o ausencia de aflatoxinas en la dieta, los efectos de los diferentes aluminosilicatos y la interacción de estos dos factores. Ya que la interacción fue significativa para varios parámetros, los resultados se sometieron a un análisis de varianza de una vía entre dietas experimentales, seguido por pruebas de rangos múltiples de Tukey ( $\alpha=0.05$, SSPS 16.0, 2007, SPSS Inc., Chicago, Illinois). 
Table 2. Percentage of water absorption capacity (WA) and dry matter loss (DML) in the experimental diets (CD, aflatoxincontaminated diet; NCD, non-contaminated diet; mean \pm standard deviation). Different letters in the same column indicate significant differences at $P<0.05$.

Tabla 2. Porcentaje de capacidad de absorción de agua (WA) y pérdida de materia seca (DML) en las dietas experimentales (CD, dieta contaminada con aflatoxinas; NCD, dieta no contaminada; media \pm desviación estándar). Las letras diferentes en la misma columna indican diferencias significativas a $P<0.05$.

\begin{tabular}{lcc}
\hline Experimental diet & WA (\%) & DML (\%) \\
\hline CD & $99 \pm 2^{\mathrm{a}}$ & $10.2 \pm 0.1^{\mathrm{d}}$ \\
CD + Novasil Plus & $111 \pm 2^{\mathrm{cde}}$ & $8.1 \pm 0.3^{\mathrm{a}}$ \\
CD + Zeolex Extra & $115 \pm 4^{\mathrm{de}}$ & $13.2 \pm 0.7^{\mathrm{f}}$ \\
CD + Mycofix Select & $109 \pm 1^{\mathrm{cd}}$ & $8.4 \pm 0.3^{\mathrm{ab}}$ \\
CD + Fixat & $116 \pm 1^{\mathrm{e}}$ & $8.6 \pm 0.2^{\mathrm{abc}}$ \\
NCD & $99 \pm 2^{\mathrm{a}}$ & $10.3 \pm 0.1^{\mathrm{e}}$ \\
NCD + Novasil Plus & $101 \pm 1^{\mathrm{ab}}$ & $10.4 \pm 0.1^{\mathrm{e}}$ \\
NCD + Zeolex Extra & $105 \pm 5^{\mathrm{abc}}$ & $9.3 \pm 0.2^{\mathrm{bc}}$ \\
NCD + Mycofix Select & $114 \pm 1^{\mathrm{de}}$ & $8.9 \pm 0.4^{\mathrm{abc}}$ \\
NCD + Fixat & $106 \pm 2^{\mathrm{bc}}$ & $9.4 \pm 0.3^{\mathrm{cd}}$ \\
SEM & 1.1 & 0.33 \\
One-way ANOVA probability & $0.000^{* *}$ & $0.000^{* *}$ \\
Factorial probability & & \\
Contamination & $0.000^{* *}$ & 0.699 \\
Test products & $0.000^{* *}$ & $0.000^{* *}$ \\
Interaction & $0.000^{* *}$ & $0.000^{* *}$ \\
\hline
\end{tabular}

** Highly significant probability $(P<0.01)$.

differences with control diets being significant in all but two cases. In contrast, no significant differences were observed between the two control diets. Dry matter loss was not affected by the inclusion of contaminated grains $(P=0.699)$; however, the test product factor and its interaction with contamination significantly affected this parameter $(P=0.000)$. The results from one-way ANOVA showed that the addition of Novasil Plus, Mycofix Select, and Fixat to CD and the addition of Zeolex Extra and Mycofix Select to NCD resulted in slightly lower dry matter loss than the rest of the test diets.

\section{Weight gain, feed intake, feed conversion ratio, survival, and nitrogen retention}

Average body weight, feed intake, growth rate, feed conversion ratio, survival, and nitrogen retention efficiency after 42 days of experimentation are presented in table 3. Feed intake was significantly affected $(P<0.001)$ by the inclusion of the contaminated grains and test products. There was significant interaction $(P<0.001)$ between these two factors. One-way ANOVA showed that CD + Zeolex Extra, NCD,

\section{Resultados}

\section{Dietas experimentales}

La composición química de las dietas experimentales fue similar entre tratamientos (tabla 1). La concentración de aflatoxinas en la CD fue alrededor de $75 \mu \mathrm{g} \mathrm{kg}^{-1}$, siendo B1 la aflatoxina predominante $\left(60.7 \mu \mathrm{g} \mathrm{kg}^{-1}\right)$, seguida por $\mathrm{B} 2$ $\left(9.7 \mu \mathrm{g} \mathrm{kg}^{-1}\right)$ y $\mathrm{G} 1\left(4.3 \mu \mathrm{g} \mathrm{kg}^{-1}\right)$. La concentración de micotoxinas en la NCD estuvo por debajo del límite de detección. El deoxinivalenol, las fumonisinas B1, B2 y B3, la ocratoxina A y la toxina T-2 estuvieron por debajo del límite de detección en las CDs.

En la tabla 2 se presentan los datos de la pérdida de materia seca y la capacidad de absorción de agua para las dietas experimentales. La absorción de agua mostró diferencias significativas en cuanto a la contaminación $(P=0.000)$, productos experimentales $(P=0.000)$ e interacción $(P=0.000)$. Ya que la interacción fue significativa, no se consideró la interpretación de los efectos principales y se realizó un análisis de varianza de una vía entre tratamientos; los resultados muestran que los aluminosilicatos incrementaron su absorción de agua, especialmente en las CDs, las diferencias con las dietas control siendo significativas en todos menos dos de los casos. En contraste, no se observaron diferencias significativas entre las dos dietas control. La pérdida de materia seca no resultó afectada por la inclusión de granos contaminados $(P=0.699)$; sin embargo, el factor del producto experimental y su interacción con la contaminación afectó significativamente este parámetro $(P=0.000)$. Los resultados del análisis de varianza de una vía muestran que la adición de Novasil Plus, Mycofix Select y Fixat a la CD y la adición de Zeolex Extra y Mycofix Select a la NCD resultó en una pérdida de materia seca ligeramente menor que en las demás dietas experimentales.

\section{Aumento de peso, consumo de alimento, tasa de conversión alimenticia, supervivencia y retención de nitrógeno}

En la tabla 3 se presentan el peso corporal promedio, el consumo de alimento, la tasa de crecimiento, la tasa de conversión alimenticia, la supervivencia y la eficiencia de retención de nitrógeno después de 42 días del experimento. El consumo de alimento resultó significativamente afectado $(P<0.001)$ por la inclusión de granos contaminados en los productos experimentales. Se observó una interacción significativa $(P<0.001)$ entre estos dos factores. El análisis de varianza de una vía mostró que $\mathrm{CD}+$ Zeolex Extra, NCD y NCD + Novasil Plus tuvieron los mayores valores de consumo de alimento (4.62-4.90 vs 3.84-4.16 g camarón $^{-1}$ para las demás dietas). La tasa de crecimiento resultó significativamente afectada $(P=0.001)$ por la inclusión de granos contaminados, mientras que los productos experimentales no modificaron este parámetro $(P>0.05)$. La tasa de 
and NCD + Novasil Plus displayed the highest feed intake (4.62-4.90 vs 3.84-4.16 $\mathrm{g} \mathrm{shrimp}^{-1}$ for the rest of the test diets). Growth rate was significantly affected by the inclusion of contaminated grains $(P=0.001)$, whereas the test products did not modify this parameter $(P>0.05)$. A significant interaction was found for growth rate. Results from one-way ANOVA indicated that shrimp fed CD + Zeolex Extra, NCD, NCD + Novasil Plus, NCD + Mycofix Select, and NCD + Fixat presented the highest growth rates $(545-620 \%$ vs $460-515 \%$ for the other diets). Feed conversion ratio and survival were significantly affected $(P<0.05)$ by the inclusion of the test products but not by the inclusion of contaminated grains. No significant interaction was found for these parameters. Shrimp fed diets supplemented with the test products (except Zeolex Extra) displayed a slightly better feed conversion ratio than shrimp fed the unsupplemented CD or NCD (1.34 vs 1.42). Shrimp fed CD + Mycofix Select and NCD + Mycofix Select showed lower survival, whereas shrimp fed CD and NCD and supplemented with Fixat, Zeolex Extra, and Novasil Plus presented a higher survival rate $(83 \%$ vs $95-98 \%)$. Nitrogen retention efficiency was not affected by the inclusion of the contaminated grains or test products, and ranged from $40 \%$ to $47 \%$ among test diets.

\section{DISCUSSION}

\section{Experimental diets}

Bentonites have been used in animal feeds to increase the efficiency of the feed manufacturing process and to enhance pellet quality by decreasing porosity in pelleted feed and reducing manufacturing costs (Thomas et al. 1998). Pfost and Young (1973) found that the inclusion of sodium bentonite had beneficial effects on pellet durability, on average 5\% higher than for control diets; however, no significant difference was observed for the use of bentonite in relation to particle size (fine, medium, coarse) of the feed ingredients. In the present study, diet stability in CDs and NCDs was significantly improved by the inclusion of Mycofix Select and Fixat (about 12-16\%); on the contrary, the addition of Novasil Plus and Zeolex Extra did not show positive results. For instance, Novasil Plus improved the stability of CD but increased the dry matter loss when added to NCD. This result is difficult to explain; however, the presence of montmorillonite and vermiculite in the clays can increase the water absorption capacity, resulting in the expansion and contraction of the mineral at molecular level (Vaught et al. 2006). Moreover, other factors such as particle and pore-size distribution, texture, specific surface area, cation exchange capacity, organic matter, and exchangeable cations may vary the water absorption (Vaught et al. 2006). Higher water absorption capacity observed in the diets supplemented with Mycofix Select and Fixat confirmed the presence of montmorillonite clay. crecimiento mostró una interacción significativa. Los resultados del análisis de varianza indicaron que los camarones alimentados con CD + Zeolex Extra, NCD, NCD + Novasil Plus, NCD + Mycofix Select y NCD + Fixat presentaron las mayores tasas de crecimiento (545-620\% vs $460-515 \%$ para las otras dietas). La tasa de conversión alimenticia y la supervivencia resultaron significativamente afectadas $(P<0.05)$ por la inclusión de los productos experimentales pero no por la inclusión de granos contaminados. No se encontró una interacción significativa para estos parámetros. Los camarones alimentados con dietas suplementadas con estos productos (excepto Zeolex Extra) mostraron una tasa de conversión alimenticia ligeramente mejor que los camarones alimentados con CD o NCD (1.34 vs 1.42). Los camarones alimentados con CD + Mycofix Select y NCD + Mycofix Select mostraron menor supervivencia, mientras que los camarones proporcionados CD y NCD y las dietas suplementadas con Fixat, Zeolex Extra y Novasil Plus presentaron una mayor tasa de supervivencia ( $83 \%$ vs $95-98 \%)$. La eficiencia de retención de nitrógeno no resultó afectada por la inclusión de granos contaminados o los productos experimentales, variando de $40 \%$ a $47 \%$ entre las dietas experimentales.

\section{Discusión}

\section{Dietas experimentales}

Las bentonitas han sido utilizadas en alimentos para animales para incrementar la eficiencia del proceso de fabricación y para mejorar la calidad del alimento reduciendo la porosidad de los alimentos peletizados y disminuyendo los costos de elaboración (Thomas et al. 1998). Pfost y Young (1973) encontraron que la inclusión de bentonita de sodio tiene efectos beneficiales en la durabilidad del alimento, en promedio $5 \%$ más alta que para dietas control; sin embargo, no se observó una diferencia significativa en el uso de la bentonita en relación con el tamaño de partícula (fino, medio, grueso) de los ingredientes alimenticios. En el presente estudio, la estabilidad de las CDs y NCDs mejoró significativamente con la inclusión de Mycofix Select y Fixat (12-16\%); al contrario, la inclusión de Novasil Plus y Zeolex Extra no produjo resultados positivos. Por ejemplo, Novasil Plus mejoró la estabilidad de la $\mathrm{CD}$ pero incrementó la pérdida de materia seca de la NCD. Este resultado es difícil de explicar; sin embargo, la presencia de montmorillonita y vermiculita en las arcillas puede aumentar la capacidad de absorción de agua, resultando en la expansión y contracción del mineral a nivel molecular (Vaught et al. 2006). Asimismo, otros factores como la distribución de partículas y tamaño de poro, textura, área superficial específica, capacidad de intercambio catiónico, materia orgánica y cationes intercambiables pueden hacer variar la absorción de agua (Vaught et al. 2006). La mayor capacidad de absorción de agua observada en las dietas suplementadas con Mycofix Select y Fixat confirmó la presencia de montmorillonita. 
Table 3. Growth performance parameters (mean \pm standard deviation, $n=3)$ of white shrimp fed experimental diets $(\mathrm{CD}$, aflatoxincontaminated diet; NCD, non-contaminated diet) for 42 days. Different letters in the same column indicate significant differences at $P<0.05$.

Tabla 3. Parámetros de crecimiento (media \pm desviación estándar, $n=3$ ) de camarón blanco alimentado con dietas experimentales $(\mathrm{CD}$, dieta contaminada con aflatoxinas; NCD, dieta no contaminada) durante 42 días. Las letras diferentes en la misma columna indican diferencias significativas a $P<0.05$.

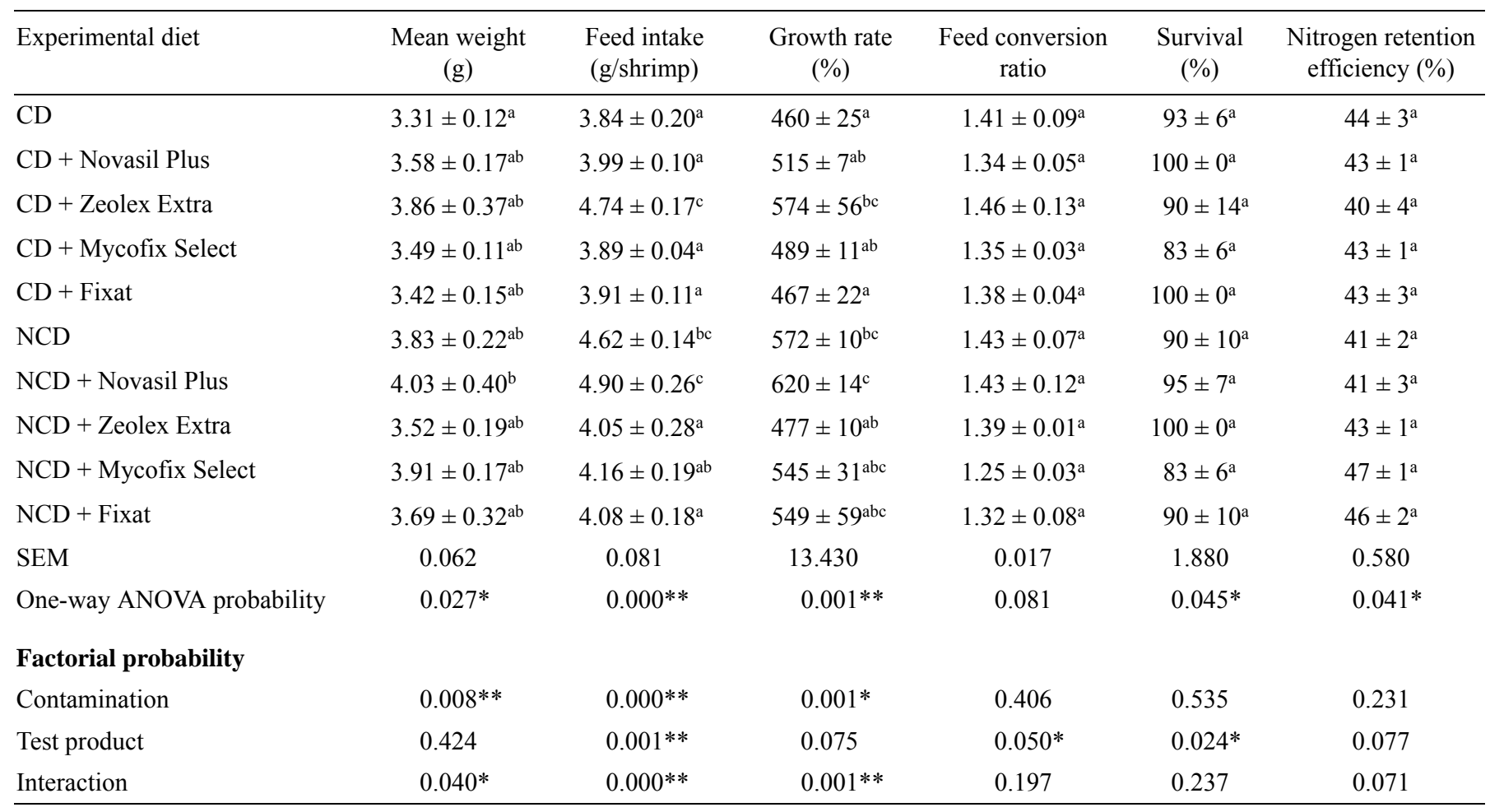

* Significant probability $(P<0.05)$.** Highly significant probability $(P<0.01)$.

\section{Safe inclusion of aluminosilicates in shrimp diets}

In terrestrial animals, a notable improvement in weight gain was observed when aluminosilicates were supplemented to uncontaminated diets and this result may be attributed to an increase in appetite, digestion, caloric efficiency, etc. (Mumpton and Fishman 1977). On the other hand, some authors have reported a reduction in body weight in terrestrial animals fed mycotoxin-free diets containing activated charcoal, diatomite (Denli and Okan 2006), or HSCAS (Döll et al. 2005). In rainbow trout, an enhancement in weight gain and feed conversion has been reported when fed uncontaminated diets supplemented with sodium bentonite or mordenite (Eya et al. 2008) or Cuban zeolites (Lanari et al. 1996), while other studies have shown negative effects. For example, Reinitz (1984) concluded that high inclusion levels of sodium bentonite in rainbow trout diets reduced weight gain and caused a disruption of the kidney tubules. Differences between results might depend on the type of zeolite used, its degree of purity, physical and chemical characteristics, and dietary concentration (Macháček et al. 2010). In the present study, Pacific white shrimp fed NCDs supplemented with Zeolex Extra, Mycofix Select, and Fixat reduced feed intake (about $10-12 \%$ ) and growth rate (about 4-17\%); in contrast,

\section{Uso de aluminosilicatos en dietas para camarones}

Se observó una mejoría importante en el aumento de peso de animales terrestres cuando aluminosilicatos fueron incluidos en dietas no contaminadas, atribuyéndose este resultado a un aumento de apetito, digestión, eficiencia calórica, etc. (Mumpton y Fishman 1977). Por otro lado, algunos autores observaron una reducción del peso corporal de animales terrestres alimentados con dietas libres de micotoxinas que contenían carbón activado, diatomita (Denli y Okan 2006) o HSCAS (Döll et al. 2005). En el caso de la trucha arcoiris, se observó mayor aumento de peso y conversión de alimento cuando se alimentó con dietas no contaminadas suplementadas con bentonita de sodio o mordenita (Eya et al. 2008) o zeolita cubana (Lanari et al. 1996), mientras que otros estudios han documentado efectos negativos. Por ejemplo, Reinitz (1984) concluyó que altos niveles de inclusión de bentonita de sodio en dietas para la trucha arcoiris disminuía el aumento de peso y dañaba los túbulos renales. Las diferencias entre los resultados pueden atribuirse al tipo de zeolita utilizado, su grado de pureza, características físicas y químicas y concentración dietética (Macháček et al. 2010). En el presente estudio, los individuos de L. vannamei alimentados con NCD + Zeolex Extra, NCD + Mycofix Select y NCD + 
shrimp fed NCD + Novasil Plus showed higher feed intake (about 6\%) and growth rate (about 8\%). It is possible that the reduction in feed intake observed with some of the test products was due to the ability of some zeolites to bind nitrogen compounds (Nguyen and Tanner 1998); shrimp respond well to the presence of attractant compounds to ensure feed intake (Suresh et al. 2011).

Positive results regarding nitrogen retention due to clay supplementation to uncontaminated diets have been reported for terrestrial animals and explained by changes in the physical properties of the diets, such as water holding capacity and swelling rate; as a result, nutrient retention may improve (Damiri et al. 2011). In the present study, a significant correlation was observed between feed intake $(\mathrm{Y}=$ $0.0069 \mathrm{X}^{2}-1.5119 \mathrm{X}+87.037 ; R^{2}=0.68$ ) or nitrogen retention efficiency $\left(\mathrm{Y}=-0.0184 \mathrm{X}^{2}+4.3746 \mathrm{X}-211.94, R^{2}=\right.$ 0.87 ) and water absorption capacity of the NCDs.

\section{Dietary aflatoxin}

The adverse effects on feed intake and growth observed in shrimp fed aflatoxin-contaminated diets are similar to those previously reported for other shrimp species (Boonyaratpalin et al. 2001, Soonngam and Hutacharoen 2007, Tapia-Salazar et al. 2012). Reduction in feed intake might be caused by the presence of non-palatable compounds generated in the grain during mold growth. No reduction in survival was found in the present study using low dietary aflatoxin levels, which is in accordance with that reported by Tapia-Salazar et al. (2012) for L. vannamei and by Boonyaratpalin et al. (2001) and Gopinath and Raj (2009) for P. monodon fed contaminated diets (from 15 to $2500 \mu \mathrm{g}$ aflatoxin $\mathrm{kg}^{-1}$ diet).

\section{Effectiveness of aluminosilicates to bind aflatoxin in shrimp diets}

In vitro and in vivo studies have shown that aluminosilicates have the capacity to bind mycotoxins (MarroquínCardona et al. 2009); however, their effectiveness depends on several factors such as absorption ability, which in turn depends on their structure and chemical components, particle size, binding capacity, exposed surface area, and physicochemical properties of the mycotoxins (Kolossova et al. 2009). For rainbow trout, a few studies have shown that the supplementation of sodium aluminosilicates and bentonites to aflatoxin-contaminated diets reduces intestinal aflatoxin absorption (Ellis et al. 2000, Hassan et al. 2010); nevertheless, other studies have shown that the addition of aluminosilicates to aflatoxin-contaminated diets for catfish and rainbow trout did not ameliorate their toxic effect (Lopes et al. 2009, Arana et al. 2011). Suppadit et al. (2006) and Soonngam and Hutacharoen (2007) observed that adding HSCAS or vermiculite to aflatoxin-contaminated diets lessened the negative effects triggered by the mycotoxin in
Fixat presentaron una disminución en su consumo de alimento (alrededor de 10-12\%) y tasa de crecimiento (alrededor de 4-17\%); en contraste, los individuos alimentados con $\mathrm{NCD}+$ Novasil Plus mostraron un mayor consumo de alimento (alrededor de 6\%) y tasa de crecimiento (alrededor de $8 \%$ ). Es posible que tal disminución del consumo de alimento se debió a la capacidad de algunas zeolitas de secuestrar compuestos de nitrógeno (Nguyen y Tanner 1998); los camarones responden bien a la presencia de compuestos atrayentes para asegurar su ingesta de alimento (Suresh et al. 2011).

Para animales terrestres se han documentado resultados positivos en cuanto a la retención de nitrógeno debido a la inclusión de arcillas en dietas no contaminadas, los cuales se han atribuido a cambios en las propiedades físicas de las dietas, tales como la capacidad de retención de agua y la tasa de hinchazón; consecuentemente, la retención de nutrientes puede mejorar (Damiri et al. 2011). En el presente estudio se observó una correlación significativa entre el consumo de alimento $\left(\mathrm{Y}=0.0069 \mathrm{X}^{2}-1.5119 \mathrm{X}+87.037 ; R^{2}=0.68\right) \mathrm{o}$ la eficiencia de retención de nitrógeno $\left(\mathrm{Y}=-0.0184 \mathrm{X}^{2}+\right.$ $4.3746 \mathrm{X}-211.94, R^{2}=0.87$ ) y la capacidad de absorción de agua de las NCDs.

\section{Aflatoxinas dietéticas}

Los efectos adversos observados en los camarones alimentados con las dietas contaminadas con aflatoxinas son similares a los documentados para otras especies de camarón (Boonyaratpalin et al. 2001, Soonngam y Hutacharoen 2007, Tapia-Salazar et al. 2012). La reducción del consumo de alimento puede estar causado por la presencia de compuestos inapetecibles generados en el grano durante el crecimiento de moho. No se observó una reducción de la supervivencia en el presente estudio usando niveles bajos de aflatoxinas, lo cual concuerda con lo documentado por Tapia-Salazar et al. (2012) para L. vannamei y por Boonyaratpalin et al. (2001) y Gopinath y Raj (2009) para P. monodon alimentados con dietas contaminadas (de 15 a $2500 \mu \mathrm{g}$ aflatoxina $\mathrm{kg}^{-1}$ dieta).

\section{Efectividad de aluminosilicatos para secuestrar aflatoxinas en dietas para camarones}

Estudios in vitro e in vivo han mostrado que los aluminosilicatos tienen la capacidad de adsorber las micotoxinas (Marroquín-Cardona et al. 2009); sin embargo, su efectividad depende de varios factores como su capacidad de absorción, la cual a su vez depende de la estructura y componentes químicos, tamaño de partícula, capacidad de unión, área superficial expuesta y propiedades fisicoquímicas de las micotoxinas (Kolossova et al. 2009). Para la trucha arcoiris, algunos estudios han mostrado que la suplementación de aluminosilicatos de sodio y bentonitas a dietas contaminadas con aflatoxinas reduce la absorción intestinal de aflatoxinas (Ellis et al. 2000, Hassan et al. 2010). Por otro lado, otros trabajos han demostrado que la inclusión de aluminosilicatos en 
L. vannamei and P. monodon, but these improvements were not statistically comparable to the uncontaminated diet. In the present study, feeding CD supplemented with Zeolex Extra to L. vannamei resulted in feed intake and growth rate similar to that of the shrimp fed NCD; in contrast, the addition of Novasil Plus, Mycofix Select, and Fixat to CD slightly improved the weight gain but failed to produce a significant feed intake recovery.

During digestion, factors like $\mathrm{pH}$, feed composition, gastrointestinal transit conditions, intestinal microflora in the gastrointestinal tract, etc., can affect mycotoxin binding to feed additives (Jaynes and Zartman 2011). Juan-Juan et al. (2010) tested the ability of three different absorbents for aflatoxin under in vitro conditions (alkaline, acidic and simulated gastric fluids; incubated for $1 \mathrm{~h}$ at $37^{\circ} \mathrm{C}$ ) and concluded that the highest affinity for aflatoxin B1 was observed under alkaline conditions. Marroquín-Cardona et al. (2009) determined the isotherms for aflatoxin $\mathrm{B} 1$ adsorption $(\mathrm{pH} 2$ and 6; incubation for $2 \mathrm{~h}$ at $25^{\circ} \mathrm{C}$ ) in 13 different additives distributed in Mexico (Milbond-TX, Mycoad, Volclay FD181, Fixat, Toxinor, Mexsil, Mycosil, Klinsil, Zeotek, Duotek, Mycosorb, Mycofix Plus 3.0, and Novasil Plus 3.0). Results showed that the most effective additives for aflatoxin B1 at both $\mathrm{pH}$ conditions (2 and 6) were Novasil Plus, MilbondTX, Mycoad, and Volclay, while the least effective was Mycosorb. In the present study, the inclusion of Zeolex Extra in CD significantly improved the feed intake and growth, whereas the other products did not produce statistically significant improvements.

Feed conversion ratio and nitrogen retention efficiency were not significantly modified by the aflatoxin-contaminated diet nor by the inclusion of aluminosilicates in the present study. Feed conversion is the ratio of feed intake and weight gain; the inclusion of contaminated grains in CD proportionally reduced feed consumption and weight gain. Aluminosilicate inclusion in the experimental diets did not significantly affect survival; this result is in agreement with that reported by Soonngam and Hutacharoen (2007) for P. monodon fed diets containing aluminosilicates. Although the aluminosilicates evaluated in the present study reduced feed intake they did not affect any other biological parameter; therefore, it is safe to use these products in diets for Pacific white shrimp.

It can thus be concluded that the inclusion of aflatoxincontaminated grains in shrimp diets reduces feed intake and weight gain. Novasil Plus, Zeolex Extra, Mycofix Select, and Fixat are safe and can be used in formulated diets for Pacific white shrimp. The aluminosilicates evaluated in the present study showed some improvement in the performance of juvenile Pacific white shrimp fed CDs; however, not all the products were able to retrieve the growth rate found for shrimp fed NCDs. More studies are needed to determine if higher doses of aluminosilicates can completely overcome the adverse effects of dietary aflatoxin on Pacific white shrimp. dietas contaminadas con aflatoxinas para bagres y trucha arcoiris no produjo una disminución de sus efectos tóxicos (Lopes et al. 2009, Arana et al. 2011). Suppadit et al. (2006) y Soonngam y Hutacharoen (2007) observaron que la inclusión de HSCAS o vermiculita en dietas contaminadas con aflatoxinas aminoraba los efectos negativos producidos por las micotoxinas en $L$. vannamei y $P$. monodon, pero estas mejorías no eran estadísticamente comparables con la dieta sin aflatoxina. En el presente estudio, el consumo de alimento y la tasa de crecimiento fueron similares para los camarones alimentados con CD + Zeolex y NCD; en contraste, la adición de Novasil Plus, Mycofix Select y Fixat a la CD mejoró ligeramente el aumento de peso, pero no mejoró significativamente el consumo de alimento.

Durante la digestión, factores como el pH, la composición alimenticia, las condiciones del tránsito gastrointestinal, la microflora intestinal en el tracto gastrointestinal, etc., pueden afectar la unión de las micotoxinas a los aditivos alimenticios (Jaynes y Zartman 2011). Juan-Juan et al. (2010) examinaron la capacidad de tres absorbentes de aflatoxina en condiciones in vitro (jugos gástricos simulados, ácidos y alkalinos; incubación durante $1 \mathrm{~h} \mathrm{a} 37^{\circ} \mathrm{C}$ ) y concluyeron que la mayor afinidad por aflatoxina B1 se observó en condiciones alkalinas. Marroquín-Cardona et al. (2009) determinaron las isotermas para la adsorción de aflatoxina $\mathrm{B} 1$ ( $\mathrm{pH} 2$ y 6; incubación durante $2 \mathrm{~h}$ a $25^{\circ} \mathrm{C}$ ) en 13 diferentes aditivos distribuidos en México (Milbond-TX, Mycoad, Volclay FD181, Fixat, Toxinor, Mexsil, Mycosil, Klinsil, Zeotek, Duotek, Mycosorb, Mycofix Plus 3.0 y Novasil Plus 3.0). Los resultados demostraron que para la aflatoxina $\mathrm{B}$ los aditivos más efectivos en ambas condiciones de $\mathrm{pH}(2 \mathrm{y} 6)$ fueron Novasil Plus, Milbond-TX, Mycoad y Volclay, mientras que el menos efectivo fue Mycosorb. En el presente estudio, la adición de Zeolex Extra a la CD mejoró significativamente el consumo de alimento y la tasa de crecimiento, mientras que los demás productos no produjeron mejorías estadísticamente significativas.

La tasa de conversión alimenticia y la eficiencia de retención de nitrógeno no resultaron significativement modificadas por la dieta contaminada con aflatoxinas ni por la inclusión de aluminosilicatos. La conversión alimenticia es la razón de consumo de alimento y aumento de peso; la inclusión de granos contaminados en la CD redujo proporcionalmente el consumo de alimento y el aumento de peso. La inclusión de aluminosilicatos en las dietas experimentales no afectó significativamente la supervivencia; este resultado concuerda con lo documentado por Soonngam y Hutacharoen (2007) para P. monodon alimentado con dietas que incluían aluminosilicatos. Aunque los aluminosilicatos evaluados en el presente estudio disminuyeron el consumo de alimento, no afectaron ningún otro parámetro biológico; por lo tanto, el uso de estos productos en dietas para el camarón blanco del Pacífico es seguro.

Se puede concluir que la inclusión de granos contaminados con aflatoxinas en dietas elaboradas para camarones 


\section{ACKNOWLEDGEMENTS}

This research was funded by the Ministry of Education and the National Council for Science and Technology (SEPCONACYT, Mexico) and by the University of Nuevo León (PAICyT-UANL, Mexico). We thank the shrimp hatchery (Langostinos y Camarones) in Boca del Río, Veracruz, for providing the shrimp for the growth trial, and Nutek Company (Tehuacán, Puebla) for providing the contaminated corn.

\section{REFERENCES}

Arana S, Dagli MLZ, Sabino M, Tabata YA, Rigolino MG, Hernández-Blázquez FJ. 2011. Evaluation of the efficacy of hydrated sodium aluminosilicate in the prevention of aflatoxin induced hepatic cancer in rainbow trout. Pesqui. Vet. Bras. 31: 751-755.

http://dx.doi.org/10.1590/S0100-736X2011000900005

Boonyaratpalin M, Supamattaya K, Verakunpiriya V, Supraser D. 2001. Effects of aflatoxin B1 on growth performance, blood components, immune function and histopathological changes in black tiger shrimp (Penaeus monodon Fabricius). Aquacult. Res. 32: 388-398. http://dx.doi.org/10.1046/j.1355-557x.2001.00046.x

Damiri H, Chaji M, Bojarpour M, Mamuei M. 2011. Effect of different sodium bentonite levels on performance, carcass traits and passage rate of broilers. Pak. Vet J. 32: 197-200.

Denli M, Okan F. 2006. Efficacy of different adsorbents in reducing the toxic effects of aflatoxin B1 in broiler diets. S. Afr. J. Anim. Sci. 36: 222-228.

Döll S, Gericke S, Dänicke S, Raila J, Ueberschär HK, Valenta H, Schnurrbusch U, Schweigert FJ, Flachowsky G. 2005. The efficacy of a modified aluminosilicate as a detoxifying agent in Fusarium toxin contaminated maize containing diets for piglets. J. Anim. Physiol. Anim. Nutr. 89: 342-358. http://dx.doi.org/10.1111/j.1439-0396.2005.00527.x

Döll, S, Baardsen G, Muller P, Koppe W, Stubhaug I, Danicke S. 2010. Effects of increasing concentrations of mycotoxins deoxynivalenol, zearalenone or ochratoxin A in diets for Atlantic salmon (Salmo salar) on growth performance and health. 14th International Symposium on Fish Nutrition and Feeding, Qingdao, China.

Ellis RW, Clements M, Tibbetts A, Winfree R. 2000. Reduction of the bioavailability of $20 \mathrm{mg} / \mathrm{kg}$ aflatoxin in trout feed containing clay. Aquaculture 183: 179-188. http://dx.doi.org/10.1016/S0044-8486(99)00292-6

Eya JC, Parsons A, Haile I, Jagidi P. 2008. Effects of dietary zeolites (bentonite and mordenite) on the performance of juvenile rainbow trout Onchorhynchus myskis. Aust. J. Bas. Appl. Sci. 2: 961-967.

Gopinath R, Raj RP. 2009. Histological alterations in the hepatopancreas of Penaeus monodon Fabricius (1798) given aflatoxin B1-incorporated diets. Aquacult. Res. 40: 1235-1242. http://dx.doi.org/10.1111/j.1365-2109.2009.02207.x

Hassan AM, Kenawy AM, Abbas WT, Abdel-Wahhab MC. 2010. Prevention of cytogenetic, histochemical and biochemical alterations in Oreochromis niloticus by dietary supplement of sorbent materials. Ecotoxicol. Environ. Saf. 73: 1890-1895. http://dx.doi.org/10.1016/j.ecoenv.2010.07.041

Jaynes WF, Zartman RE. 2011. Aflatoxin toxicity reduction in feed by enhanced binding to surface-modified clay additives. Toxins, 3: $551-565$.

http://dx.doi.org/10.3390/toxins3060551 reduce el consumo de alimento y el aumento de peso. El uso de Novasil Plus, Zeolex Extra, Mycofix Select y Fixat es seguro y estos productos pueden incluirse en dietas formuladas para el camarón blanco del Pacífico. Los aluminosilicatos evaluados mejoraron ligeramente el desarrollo de los juveniles de L. vannamei alimentados con las CDs; sin embargo, no todos los productos pudieron proporcionar la tasa de crecimiento de los camarones alimentados con las NCDs. Se requieren estudios adicionales para determinar si dosis más altas de aluminosilicatos pueden superar completamente los efectos adversos de las aflatoxinas en las dietas para el camarón blanco del Pacífico.

\section{Agradecimientos}

Este trabajo fue apoyado por la Secretaría de Educación Pública y el Consejo Nacional de Ciencia y Tecnología (SEPCONACYT, México) y por la Universidad de Nuevo León (PAICyT-UANL, México). Agradecemos al Laboratorio de Langostinos y Camarones (Boca del Río, Veracruz) y la empresa Nutek (Tehuacán, Puebla) el haber proporcionado los camarones y el grano contaminado, respectivamente.

Traducido al español por Christine Harris.

Juan-Juan LI, Suo De-Ch., Su XO. 2010. Binding capacity for aflatoxin B1 by different adsorbents. Agric. Sci. China 9(3): 449-456. http://dx.doi.org/10.1016/S1671-2927(09)60116-4

Kolossova A, Stroka J, Breidbach A, Kroeger K. 2009. Evaluation of the effect of mycotoxin binders in animal feed on the analytical performance of standardized methods for the determination of mycotoxins in feed. JCR Scientific and Technical Reports 54375. Luxembourg, Office for Official Publications of the European Communities, $49 \mathrm{pp}$. http://dx.doi.org/10.2787/15352

Lanari DD, Agaro E, Turri C. 1996. Use of Cuban zeolites in trout diets. Riv. Ital. Acquacol. 31: 23-33.

Lopes PRS, Pouey JLOF, Enke DBS, Mallmann CA, Kich HA, Soquetta MB. 2009. Utilização de adsorvente em rações contendo aflatoxina para alevinos de jundiá. Rev. Bras. Zootec. 38: 589-595. http://dx.doi.org/10.1590/S1516-35982009000400001

Macháèek M, Veèerek V, Mas N, Suchý P, Straková E, Šerman V, Herzig I. 2010. Effect of the feed additive clinoptilolite (ZeoFeed) on nutrient metabolism and production performance of laying hens. Acta Vet. Brno 79: S29-S34. http://dx.doi.org/10.2754/avb201079S9S029

Marroquín-Cardona A, Deng Y, Taylor JF, Hallmark CT, Johnson NM, Phillips TD. 2009. In vitro and in vivo characterization of mycotoxin-binding additives used for animal feeds in Mexico. Food Addit. Contam. Part A Chem. Anal. Control Expo. Risk Assess. 26: 733-743. http://dx.doi.org/10.1080/02652030802641872

Mohapatra S, Sahu NP, Pal AK, Prusty AK, Kumar V, Kumar S. 2011. Haemato-immunology and histo-architectural changes in Labeo rohita fingerlings: Effect of dietary aflatoxin and mould inhibitor. Fish Physiol. Biochem. 37: 177-186. http://dx.doi.org/10.1007/s10695-010-9428-1 
Mumpton FA, Fishman PH. 1977. The application of natural zeolites in animal science and aquaculture. J. Anim. Sci. 45: 1188-1203.

Nguyen ML, Tanner CC. 1998. Ammonium removal from wastewaters using natural New Zealand zeolites. N. Z. J. Agric. Res. 41: 427-446. http://dx.doi.org/10.1080/00288233.1998.9513328

Pfost HB, Young LR. 1973. Effect of colloidal binders and other factors on pelleting. Feedstuffs 45: 21-22.

Piva G, Galvano F, Pietri A, Piva A. 1995. Detoxification methods of aflatoxins. A review. Nutr. Res. 15: 767-776. http://dx.doi.org/10.1016/0271-5317(95)00042-H

Ramos AJ, Hernández E. 1996. In vitro aflatoxin adsorption by means of a montmorillonite silicate. A study of adsorption isotherms. Anim. Feed Sci. Technol. 62: 263-269. http://dx.doi.org/10.1016/S0377-8401(96)00968-6

Reinitz G. 1984. The effect of nutrient dilution with sodium bentonite in practical diets for rainbow trout. Prog. Fish Cult. 46: $249-253$.

http://dx.doi.org/10.1577/1548-8640(1984)46<249:TEONDW > 2.0.CO;2

Schell TC, Lindemann MD, Kornegay ET, Blodgett DJ, Doerr JA. 2000. Effectiveness of different types of clay for reducing the detrimental effects of aflatoxin-contaminated diets on performance and serum profiles of weanling pigs. J. Anim. Sci. 71: 1226-1231.

Shehata SA, El-Melegy KM, Ebrahim MS. 2009. Toxicity reduction of aflatoxin B1 by vitamin C in fish. J. Arab. Aquacult. Soc. 4: $73-86$.
Soonngam CAL, Hutacharoen R. 2007. Vermiculite and hydrated sodium calcium aluminosilicates as the agent of aflatoxin B1 absorption for black tiger shrimp diets. Environ. Nat. Resour. J. 5: $50-58$.

Suppadit T, Jaturasitha S, Pripwai N. 2006. Utilization of hydrated sodium calcium aluminosilicate and vermiculite for aflatoxin B1 adsorption in Pacific white shrimp (Litopenaeus vannamei) diets. J. Appl. Anim. Res. 29: 129-132. http://dx.doi.org/10.1080/09712119.2006.9706587

Suresh AV, Kumaraguru-vasagam KP, Nates S. 2011. Attractability and palatability of protein ingredients of aquatic and terrestrial animal origin, and their practical value for blue shrimp, Litopenaeus stylirostris, fed diets formulated with high levels of poultry byproduct meal. Aquaculture 319 : 132-140. http://dx.doi.org/10.1016/j.aquaculture.2011.06.039

Tapia-Salazar M, García-Pérez OD, Velásquez-Soto RA, NietoLópez MG, Villarreal-Cavazos D, Ricque-Marie D, Cruz-Suárez LE. 2012. Growth, feed intake, survival, and histological response of white shrimp Litopenaeus vannamei fed diets containing grains naturally contaminated with aflatoxin. Cienc. Mar. 38: 491-504. http://dx.doi.org/10.7773/cm.v38i3.2094

Thomas M, van Vliet T, van der Poel AFB. 1998. Physical quality of pelleted animal feed 3: Contribution of feedstuff components. Anim. Feed Sci. Tech. 70: 59-78. http://dx.doi.org/10.1016/S0377-8401(97)00072-2

Vaught R, Brye KR, Miller DM. 2006. Relationships among coefficient of linear extensibility and clay fractions in expansive, stoney soils. Soil Sci. Soc. Am. J. 70: 1983-1990. http://dx.doi.org/10.2136/sssaj2006.0054

Received June 2012, received in revised form November 2012, accepted November 2012. 